\title{
Family-focused CBT is not superior to enhanced treatment-as-usual in reducing suicide attempts
}

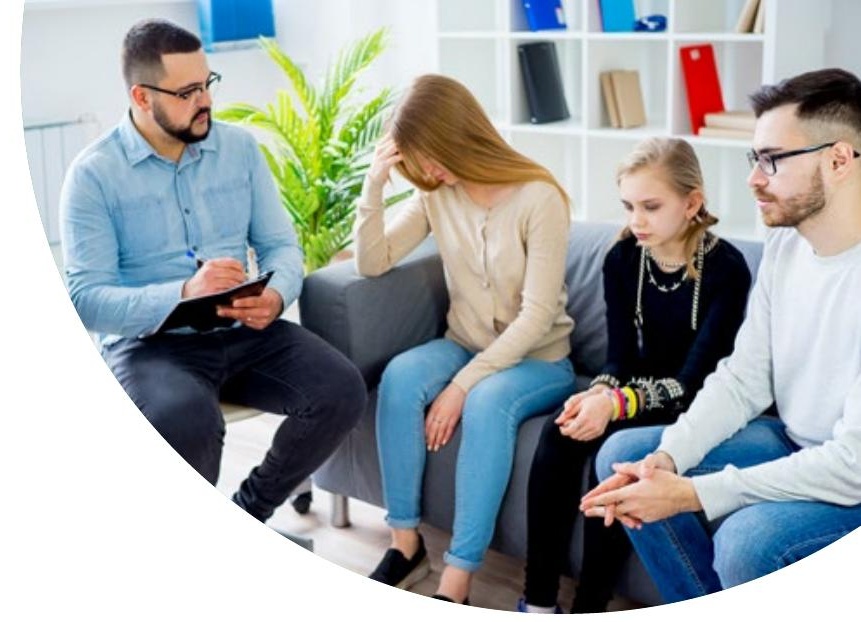

By Jessica K. Edwards

In 2011, Esposito-Smythers et al. reported that integrated outpatient cognitive-behavioural therapy (I-CBT) significantly reduced substance use, suicidal behaviours, and the rate of health service use compared with enhanced treatment-as-usual (E-TAU) in adolescents with co-occurring alcohol or drug use disorder and suicidality. I In a recent follow-up study, the researchers assessed whether a modified version of I-CBT, known as family-focused CBT (F-CBT), can also reduce the rate of suicide attempts (SA), depression, suicidal ideation (SI), or non-suicidal self injury (NSSI) in a cohort of depressed, suicidal adolescents recruited from an inpatient psychiatric hospitalization program. Although adolescents across all trial arms achieved reductions in the rates of SA, depression, SI, and NSSI over an 18-month period, the researchers found no evidence for an increased efficacy for F-CBT over E-TAU.

The researchers propose that modifications made to the F-CBT protocol, to account for differences in the sample composition, might have had a role in these differential outcomes. For example, the potential efficacy of the parent component of the intervention might have been reduced as a result of limiting the number of parent "training" sessions to accommodate for more parent "self-care" sessions. Other factors that the researchers propose might be responsible for these divergent findings include differences in the levels of substance use ( $22 \%$ vs. $100 \%)$, conduct disorder $(22 \%$ vs. $35 \%$ ) and generalized anxiety disorders (40\% vs. $16 \%$ ) in the F-CBT versus I-CBT studies. The researchers suggest that increasing the frequency of F-CBT sessions at the start of treatment might, therefore, be necessary to see an effect in this population.

Esposito-Smythers, C. et al. (2019), Family-focused cognitive-behavioural treatment for depressed adolescents in suicidal crisis with co-occurring risk factors: a randomized trial. J. Child Psychol. Psychiatr. doi:10.1111/ jcpp.13095

\section{References}

${ }^{1}$ Esposito-Smythers, C., Spirito, A., Kahler, C. W., Hunt, J., \& Monti, P. (2011). Treatment of co-occurring substance abuse and suicidality among adolescents: A randomized trial. Journal of Consulting and Clinical Psychology, 79, 728-739

\section{Glossary}

\section{Integrated outpatient cognitive behavioural} therapy (I-CBT): I-CBT protocols integrate CBT techniques to remediate maladaptive cognitions and behaviours. In the 2011 study by EspositoSmythers, et al., I I-CBT included various cognitivebehavioural individual adolescent (e.g., problemsolving, refusal skills), family (e.g., communication, behavioural contracting) and parent training (e.g., monitoring, emotion regulation) sessions. Motivational interviewing sessions for adolescents to improve readiness for treatment and for parents to facilitate treatment engagement were also provided. The sessions could be repeated and practiced throughout the protocol, and, casemanagement calls were made as needed outside of sessions.

\section{Family-focused outpatient cognitive} behavioural therapy (F-CBT): The F-CBT protocol used by Esposito-Smythers, et al. in 2019 was based on I-CBT,1 with modifications to better accommodate the broader sample of suicidal youth recruited to the study. Added sessions included those addressing emotion regulation (distress tolerance), physical health (healthy lifestyle), trauma (trauma narrative) and anxiety (exposure). The scope of the sessions was also broadened to be relevant to any highrisk behaviour. Parental self-care sessions were also added, in which therapists taught parents seven skills that in I-CBT were only taught to adolescents. Finally, F-CBT included a parent training emotion coaching session to improve parent-child interactions. 